DOI: 10.1136/annrheumdis-2018-eular.4040

\section{THU0624 COLCHICINE: AN EFFECTIVE TREATMENT OPTION FOR UNCLASSIFIED AUTOINFLAMMATORY DISEASES IN CHILDREN}

\author{
J.B. Kuemmerle-Deschner ${ }^{1}$, A.-L. Schock ${ }^{1}$, S. Hansmann ${ }^{1}$, S.M. Benseler ${ }^{2}$. \\ ${ }^{1}$ Department of Pediatrics, Division of Rheumatology, University Hospital \\ Tuebingen, Tuebingen, Germany, ${ }^{2}$ Rheumatology, Department of Paediatrics, \\ Alberta Children's Hospital, University of Calgary, Calgary, Alberta, Canada
}

Background: Children and adults with clinically and genetically defined autoinflammatory diseases (AID) including CAPS, TRAPS and HIDS can receive expensive Interleukin-1 (IL-1) inhibitors in many countries around the world. However, patients suffering from unclassified autoinflammatory conditions characterised by recurrent fevers and organ dysfunction and the absence of a known pathogenic mutation commonly have no access to these treatment options.

Objectives: The aim of this study was to explore the efficacy and safety of colchicine treatment in children and adults with autoinflammatory diseases without pathogenic mutations.

Methods: Consecutive children and adults with autoinflammatory diseases without pathogenic mutations treated with colchicine were included in this single centre study and observed for a median of 12,94 months (range 1,25-66,73). Clinical features, autoinflammatory disease activity indices (AIDAI), inflammatory markers ESR, CRP, SAA and S100, frequency and duration of flares and physician global assessment of disease activity (VAS) were recorded serially and compared at baseline and while receiving Colchicine therapy.

Results: A total of 39 patients were included in the study. These were 16 girls and 23 boys, median age at start of colchicine therapy was 4 years (range 1-54). The diagnoses included PFAPA in 15, mutation-negative FMF in 11, autoinflammation with low-penetrance variants in nine (all NLRP3) and other unclassified AID in four patients. Recurrent fever was the leading symptom, mostly associated with arthralgia and myalgia. The mean disease activity decreased from 4.4 at baseline to 2.2 on colchicine. Mean SAA-levels decreased from 159 to $63.3 \mathrm{mg} / \mathrm{L}$, CRP levels from 6.4 to $2.3 \mathrm{mg} / \mathrm{dl}$. Flare frequency was reduced in $72 \%$ and remained unchanged in $28 \%$ of patients. Flare duration was reduced in $82 \%$, unchanged in $14 \%$ and increased in only $4 \%$ of patients. Most common adverse events were abdominal pain and nausea in $50 \%$ of patients and appeared to be dose dependent.

Conclusions: Children and adults with unclassified autoinflammatory diseases may benefit significantly for colchicine therapy. Control of clinical disease activity and improved inflammatory markers were documented in $59 \%$ of patients. Colchicine should be considered in patients with active inflammatory disease with no access to IL-1 inhibitors. Controlled trials are needed to further explore this approach.

Disclosure of Interest: None declared

DOI: 10.1136/annrheumdis-2018-eular.7473

\section{THU0625 IGG4-RELATED DISEASE MANIFESTATIONS DIFFER BETWEEN ASIAN AND NON-ASIAN SUBJECTS}

Z.S. Wallace ${ }^{1}$, K. Okazaki ${ }^{2}$, C. Perugino ${ }^{1}$, H. Umehara ${ }^{3}$, T. Saeki ${ }^{4}$, M. Kawano ${ }^{5}$, Y. Zen ${ }^{6}$, R. Naden ${ }^{7}$, J.H. Stone ${ }^{1}$, on behalf of For the EULAR/ACR IgG4-Related Disease Classification Criteria Development Group. ${ }^{1}$ Massachusetts Gen Hosp Rheumatol Unit, Harvard Med School, Boston, USA; ${ }^{2}$ Kansai Medical University, Osaka; ${ }^{3}$ Shiritsu Nagahama Byoin, Nagahama; ${ }^{4}$ Nagaoka Red Cross Hospital, Nagaoka; ${ }^{5}$ Kanazawa University, Kanazawa; ${ }^{6}$ University of Kobe, Kobe, Japan; ${ }^{7}$ University of Hamilton, Hamilton, Canada

Background: Background: IgG4-related disease (IgG4-RD) is a multi-system immune-mediated condition that can affect nearly any organ. No study has evaluated differences in disease manifestations according to race. We evaluated this in a large cohort of IgG4-RD subjects submitted by an international group of investigators.

Objectives: Objectives: To evaluate racial differences in manifestations of IgG4$\mathrm{RD}$.

Methods: Methods: To validate the ACR/EULAR IgG4-RD Classification Criteria, 76 investigators from North America, South America, Europe, and Asia submitted cases they considered to be IgG4-RD in either the preliminary phase or the validation phase. For each case, investigators included details related to diagnostic certainty, age at disease onset and diagnosis, race, organ involvement, biopsy findings, and laboratory results. Based on reported race, we dichotomized subjects into either Asian or non-Asian categories; subjects of South Asian $(n=14)$ descent (e.g., India, Pakistan), all of whom resided in North America or Europe were grouped with non-Asian subjects. We compared the distribution of disease features according to race using t-tests, Wilcoxon tests, and Chi square tests, where appropriate, as well as in multivariable-adjusted models.
Results: Results: In the validation phase, there were 493 cases of IgG4-RD submitted by 23 investigators who practice in Asia and 29 investigators who practice in North America or Europe. There was no significant difference in the distribution of specialists (e.g., rheumatology, gastroenterology) between Asian and nonAsian investigators $(p=0.3)$. The majority of IgG4-RD subjects, both Asian $(n=208)$ and non-Asian $(n=285)$, were male $(61 \%$ and $69 \%$, respectively). Asian subjects were significantly older both at symptom onset and diagnosis $(61.2 \pm 13.2$ years and $62.6 \pm 12.8$ years, respectively) compared to non- Asian subjects (55.1 \pm 14.9 years and $57.2 \pm 14.4$ years, respectively, $p<0.0001$ for both comparisons). There was a significantly shorter diagnostic delay among Asian subjects compared to non-Asian subjects $(1.4 \pm 2.7$ years vs $2.2 \pm 3.7$ years, $p=0.01)$. Head/neck involvement was more common in Asians ( $52 \%$ vs $27 \%, p<0.0001)$ whereas hepatobiliary involvement was more common in non-Asians ( $52 \%$ vs. $42 \%, p=0.04)$ Asian subjects had a significantly higher median serum IgG4 concentration (666 mg/dL, IQR 320.5-1230 vs 240.5, IQR 100-505, p<0.0001) and were more likely to have a serum IgG4 concentration greater than five times the upper limit of normal $(48 \%$ vs $20 \%, p<0.0001)$. In multivariable-adjusted models, differences in age and serum IgG4 concentration according to race remained strongly significant ( $p<0.001$ for both comparisons).

Conclusions: Conclusions: Asian and non-Asian subjects differed regarding the age of disease onset and diagnosis, the distribution of organ involvement, and baseline serum IgG4 concentrations. There was a significantly shorter diagnostic delay among Asian subjects compared to non-Asian subjects. The etiology(ies) of these observed differences in the respective presentation of IgG4-RD in Asian and non-Asian subjects requires further investigation, but could include differences in diagnostic approach, environmental factors, and genetic predisposition.

Disclosure of Interest: None declared DOI: 10.1136/annrheumdis-2018-eular.5245

\section{THU0626 \\ CORRELATION AMONG SERUM AMYLOID A LEVELS, CLINICAL MANIFESTATIONS, TREATMENT AND DISEASE ACTIVITY IN PATIENTS WITH BEHÇET'S DISEASE}

J. Sota ${ }^{1}$, A. Vitale ${ }^{1}$, O.M. Lucherini ${ }^{1}$, R. Franceschini ${ }^{1}$, B. Frediani ${ }^{1}$, I. Orlando ${ }^{1}$, M. Galeazzi ${ }^{1}$, C. Fabiani ${ }^{2}$, G.M. Tosi ${ }^{1}$, L. Cantarini ${ }^{1}$. ${ }^{1}$ University of Siena, Siena; ${ }^{2}$ Humanitas Clinical and Research Center, Milan, Italy

Background: Behçet's disease (BD) is an inflammatory disorder potentially lead ing to life- and sight-threatening complications: no laboratory marker correlating with disease activity or predicting the occurrence of disease manifestations is currently available in the clinical practice.

Objectives: To search for a correlation between serum amyloid-A (SAA) levels and disease activity evaluated via BD current activity form (BDCAF), to assess disease activity in relationship with different SAA thresholds, to examine the association between single organ involvements and the overall major organ involvement with different SAA thresholds, and to assess the influence of biologic therapy on SAA levels.

Methods: Ninety-five serum samples were collected from 64 BD patients, and their related demographic, clinical and therapeutic data were retrospectively collected.

Results: No correlation was identified between SAA levels and BD disease activity (Spearman $r h o=0.085 ; p=0.411$ ), while a significant difference was found in the mean BDCAF score between patients presenting SAA levels $<200 \mathrm{mg} / \mathrm{L}$ and those with SAA levels $>200 \mathrm{mg} / \mathrm{L}(p=0.027)$. SAA levels higher than $200 \mathrm{mg} / \mathrm{L}$ were significantly associated with major organ involvement $(p=0.008)$. A significant association was found between SAA levels $>150 \mathrm{mg} / \mathrm{dl}$ and ocular $(p=0.008)$ skin $(p=0.002)$ and mucosal manifestations $(p=0.012)$. Patients undergoing biologic therapies were significantly associated with SAA levels $<200 \mathrm{mg} / \mathrm{L}$ compared with patients who were not treated with biologics $(p=0.012)$.

Conclusions: SAA level does not represent per se a biomarker of disease activity, but might be useful as a predictor of major organ involvement and ocular disease relapse at certain thresholds in patients with $\mathrm{BD}$.

Disclosure of Interest: None declared

DOI: 10.1136/annrheumdis-2018-eular.1178

\section{THU0627 DEMOGRAPHICS AND PRESENTING ORGAN INVOLVEMENT IN A COHORT OF PATIENTS WITH SARCOIDOSIS}

K. Sheth ${ }^{1}$, G. Jeyashanmugaraja ${ }^{2}$, J. Simard ${ }^{1}$, S. Shoor ${ }^{1} .{ }^{1}$ Stanford University, Palo Alto, USA; ${ }^{2}$ Stanley Medical College, Chennai, India

Background: Sarcoidosis is a multisystem disorder of unknown etiology charac terised pathologically by non-caseating granulomas in involved organs. Although mortality is reported in only $1 \%-5 \%$ of patients, there is data suggesting it might 
be increasing and there is little information about the clinical and epidemiological characteristics of this group of patients.

Objectives: To compare basic demographics of a cohort of deceased sarcoid patients with a non-deceased sarcoid population, and to describe the presenting organ involvement among the deceased sarcoid population.

Methods: Patients seen at the Stanford University Hospital and Clinics from 2009-2017 who were $>18$ years of age with at least one ICD-9 or 10 diagnosis of sarcoidosis and at least one clinical note with the term "sarcoidosis" were identified by EMR. After determining the demographics of this cohort, deceased patients were identified and an extensive chart review of these subset of patients was conducted. The project was approved by Stanford's Institutional Review Board.

Results: 1190 adult patients with an ICD code for sarcoidosis were identified Demographic comparison between the non-deceased and deceased population is summarised in the table 1 below.

\begin{tabular}{lcc}
\hline & Non-deceased sarcoid patients & Deceased sarcoid patients \\
\hline Total patients & 1086 & 104 \\
Gender: & $660(60.8 \%)$ & $59(56.7 \%)$ \\
Females & $426(39.2 \%)$ & $45(43.3 \%)$ \\
Males & & \\
Race: & $67(6.2 \%)$ & $1(0.9 \%)$ \\
Asian & $197(18.1 \%)$ & $26(25 \%)$ \\
Black & $596(54.9 \%)$ & $45(43.2 \%)$ \\
White & $6(0.6 \%)$ & $0(0 \%)$ \\
Native American & $128(11.8 \%)$ & $18(17.4 \%)$ \\
Others & $91(8.4 \%)$ & $14(13.5 \%)$ \\
Unknown & & \\
Ethnicity & $918(84.5 \%)$ & $80(76.9 \%)$ \\
Non-Hispanic & $67(6.2 \%)$ & $11(0.5 \%)$ \\
Hispanic/Latino & $101(9.3 \%)$ & $13(12.6 \%)$ \\
Unknown & &
\end{tabular}

In comparison with non-deceased patients with sarcoidosis, the proportion of African American population was higher in the deceased population, whereas the proportion of Asians and whites was lower in the deceased population. The percentage of male patients in the deceased subset was slightly higher than the nondeceased population. 65 (62.5\%) of the 104 deceased patients were symptomatic at diagnosis, 6 (5.8\%) were asymptomatic and information was not available for $33(31.7 \%)$ patients. Of the 65 symptomatic patients, presenting organ involvement was either pulmonary $(34,52.3 \%)$, cardiac $(11,16.9 \%)$, cutaneous $(8,12.3 \%)$, neurological(4,6.2\%), ocular(3, 4.6\%), constitutional(5,7.7\%), unknown(1,1.5\%), musculoskeletal(1,1.5\%), renal-calcium related(1,1.5\%), hepatic(2,3\%), laryngeal $(1,1.5 \%)$, nasal and sinus(2,3\%), parotid gland(1,1.5\%), gastrointestinal $(1,1.5 \%)$, or others $(5,7.6 \%)$.

Conclusions: This study compares the demographics of subset of deceased sarcoid patients with a population of non-deceased sarcoid patients, and describes the presenting organ involvement among the deceased sarcoid population. African American males had a higher mortality rate compared to Asians and Caucasians. Pulmonary and cardiac disease represented $69.2 \%$ of organ involvement in the deceased. Our study showed a mortality rate of $8.7 \%$ in the sarcoid population which is in keeping with the newer studies that mortality in patients with sarcoid might be on the rise. With further study of this population, we aim to compare the characteristics of non-deceased sarcoid patients with the deceased patients, including presenting organ involvement, diagnostic workup and treatment regimen and hope to gain a better understanding of the possible reasons for increasing mortality in patients with sarcoidosis.

Disclosure of Interest: None declared

DOI: 10.1136/annrheumdis-2018-eular.6164

\section{THU0628 DIFFERENCES IN CLINICAL MANIFESTATIONS OF BEHCET'S SYNDROME BY GENDER: CROSS- SECTIONAL ANALYSIS IN A UK COHORT}

L. Chadwick ${ }^{1}$, T. Blake ${ }^{2}$, N.J. Goodson ${ }^{1}$, D. Situnayake ${ }^{2}$, R.J. Moots ${ }^{1} .{ }^{1}$ Behcet's Syndrome Centre of Excellence, Aintree University Hospital NHS Foundation Trust, Liverpool; ${ }^{2}$ Behcet's Syndrome Centre of Excellence, Sandwell and West Birmingham NHS Foundation Trust, Birmingham, UK

Background: Geographical variations in genotype and phenotype of Behçet's syndrome (BS) are reported. ${ }^{1,2}$ Previous meta-analysis of international cohorts has highlighted differences in clinical manifestations of BS by gender. ${ }^{3} \mathrm{~A}$ comparison of BS clinical manifestations by gender has not been analysed previously in a large UK BS cohort.

Objectives: 1) To compare the clinical manifestations of BS in a UK cohort by gender and 2) to compare this to published international data.
Methods: A retrospective cross sectional analysis was performed using clinical databases at the Liverpool and Birmingham BS Centres of Excellence. Patients with a multi-disciplinary diagnosis of BS or International Study Group (ISG) diagnostic criteria were included. Clinical manifestations and HLA-B51 positivity were compared by gender. T-tests and chi-squared tests were used for continuous and categorical data respectively and a p value of 0.05 or less was considered statistically significant.

Results: 433 patients met inclusion criteria (140 males, 32.3\%). Male patients were younger and had significantly higher rates of ocular and vascular involvement, papulopustular skin rash and HLA-B51 positivity. Female patients had significantly higher rates of genital aphthosis and arthralgia.

\begin{tabular}{|c|c|c|c|}
\hline & Male $(n=140)$ & Female $(n=293)$ & $p$ value \\
\hline Age (years), mean (s.d.) ${ }^{*}$ & $41.2(12.5)$ & $44.1(11.9)$ & 0.02 \\
\hline Recurrent oral aphthosis, $n$ (\%) & $139(99.3)$ & $291(99.3)$ & 0.97 \\
\hline Genital aphthosis, $\mathrm{n}(\%)^{*}$ & $111(79.3)$ & $278(94.9)$ & $<0.01$ \\
\hline Papulopustular skin rash, $\mathrm{n}(\%)^{*}$ & $69(49.3)$ & $107(36.5)$ & 0.01 \\
\hline Erythema nodosum, $\mathrm{n}(\%)$ & $29(20.7)$ & $56(19.1)$ & 0.70 \\
\hline Skin aphthosis $(n, \%)$ & $7(5.0)$ & $8(2.7)$ & 0.23 \\
\hline Uveitis, $\mathrm{n}(\%)^{*}$ & $81(57.9)$ & $99(33.8)$ & $<0.01$ \\
\hline Retinal vasculitis, $\mathrm{n}(\%)^{*}$ & $39(27.9)$ & $36(12.3)$ & $<0.01$ \\
\hline Central nervous system involvement, $\mathrm{n}(\%)$ & $16(11.4)$ & $20(6.8)$ & 0.11 \\
\hline Large vein thrombosis, $\mathrm{n}(\%)^{*}$ & $22(15.7)$ & $19(6.5)$ & $<0.01$ \\
\hline Arterial thrombosis or aneurysm, $\mathrm{n}(\%)$ & $4(2.9)$ & $7(2.4)$ & 0.77 \\
\hline Thrombophlebitis, $\mathrm{n}(\%)^{*}$ & $10(7.1)$ & $5(1.7)$ & $<0.01$ \\
\hline Gastro-intestinal involvement, $\mathrm{n}(\%)$ & $8(5.7)$ & $25(8.5)$ & 0.30 \\
\hline Arthralgia, $\mathrm{n}(\%)^{*}$ & $68(48.6)$ & $180(61.4)$ & 0.01 \\
\hline Ethnicity (British or White), $\mathrm{n}(\%)^{*}$ & $\begin{array}{c}90(64.3) \\
(n=126)\end{array}$ & $\begin{array}{c}238(85.9) \\
(n=277)\end{array}$ & $<0.01$ \\
\hline HLA-B51 positive, $n(\%)^{*}$ & $\begin{array}{c}14(58.3) \\
(n=27)\end{array}$ & $\begin{array}{c}9(20.0) \\
(n=45)\end{array}$ & $<0.01$ \\
\hline
\end{tabular}

*statistically significant

Conclusions: Similar to published international cohorts, male patients with BS in the UK have higher rates of ocular and venous involvement, papulopustular skin rash and HLA-B51 positivity and lower rates of genital aphthosis and arthralgia in comparison to female patients. However, the extent to which these differences are attributable to ethnicity is unclear.

\section{REFERENCES :}

[1] Leonardo NM, McNeil J. Behcet's Disease: Is There Geographical Variation? A Review Far from the Silk Road. Int J Rheumatol 2015: 1-7. doi:10.1155/2015/945262

[2] Sibley C, Yazici Y, Tascilar K, Khan N, Bata Y, Yazici H, et al. Behçet syndrome manifestations and activity in the United States versus Turkey - a cross-sectional cohort comparison. J Rheumatol 2014;41:137984 doi:10.3899/jrheum.131227

[3] Bonitsis NG, Nguyen LBL, LaValley MP, Papoutsis N, Altenburg A, et al. Gender-specific differences in Adamantiades-Behçet's disease manifestations: an analysis of the German registry and meta-analysis of data from the literature. Rheumatology (Oxford). 2015;54: 121-33. doi:10.1093/rheu matology/keu247

Disclosure of Interest: None declared

DOI: 10.1136/annrheumdis-2018-eular.3249

\section{THU0629 A STUDY OF WBMRI IN ESTIMATING DISEASE ACTIVITY OF PM/DM AND THE FOLLOWING DISEASES DIAGNOSIS}

Y. Li, D. Jiang, X. Zheng, L. Lu, Z. Li, Y. Guo, J. Tang, X. He, D. Xu, G. Gao. the First Affiliated Hospital of Zhengzhou University, Zhengzhou, China

Background: IM is a group of disease characterised by chronic symmetrical myasthenia, muscle fatigue and monocyte infiltrating skeletal muscle. This disease usually associates with the muscle of trunk and proximal limbs, skin lesions companied or not. Dermatomyositis (DM) and polymyositis (PM) account for the major part of IM clinically. The diagnose of these diseases mainly rely on clinical manifestation, biopsy, ECG and serum creatase. Whole-body magnetic resonance imaging (WBMRI) has been reported succeeding in diagnosing PM/DM through short tau inversion recovery (STIR) sequence, but the ability to estimate disease activity hasn't been reported.

Objectives: To evaluate the value in WBMRI through STIR sequence in evaluating the disease activity of polymyositis (PM) and dermatomyositis (DM) and screening interstitial lung disease (ILD) and osteonecrosis. 